\title{
Task learning based on the imitation of object's goal
}

\author{
Carlos A. Acosta Calderon ${ }^{\mathrm{a}, *}$ and Rajesh E. Mohan ${ }^{\mathrm{b}}$ \\ ${ }^{a}$ School of Electrical and Electronics Engineering, Singapore Polytechnic, Singapore \\ ${ }^{\mathrm{b}}$ Singapore University of Technology and Design, Singapore
}

\begin{abstract}
Learning by imitation represents a useful and promising alternative to program robots. The approach presented here is based on two functional elements used by humans to understand and perform actions. These elements are: the body schema and the body percept. The first one is a representation of the body that contains information of the body's capabilities. The body percept is a snapshot of the body and its relation with the environment at a given instant. These elements are believed to interact within humans to generate several abilities, among them the ability to imitate. This paper presents an approach to robot imitation based on these two functional elements, which approaches imitation by identifying the goals from the perceived actions.
\end{abstract}

Keywords: Robot imitation, body representation, action representation

\section{Introduction}

Humans teach one another how to perform a task by demonstrating the required steps and actions to complete the task. In this context, human learners are capable of register a great amount of sensory data, which most of them have to be deduced from the perspective of the demonstrator. From all this pool of data the learner must selects what is relevant information to successfully complete the task. Finally in order to reproduce the task successfully, the learner must be able to extract the demonstrator's goals. Only then the learner can generalize the acquired knowledge to apply it to different situations.

The ability to imitate a task holds advantages against other learning techniques like "trial-and-error"; among these advantages imitation speeds up the learning process, one demonstrator can teach many learners. Researchers are adopting this type of learning due to

*Corresponding author: Carlos A. Acosta Calderon, School of Electrical and Electronics Engineering, Singapore Polytechnic, 500 Dover Road, 139651 Singapore. E-mail: CarlosAcosta@sp.edu.sg. its advantages; imitation is seen as a promising way to program robots, in particular with the idea in mind that robots will be operating in different environments and that they will require learning different task according to the user needs $[1,8]$. Traditional approaches to robot programming, such as traditional programming and learning strategies, have been demonstrated to be complex, slow and restricted in knowledge. Learning from demonstration would greatly reduce the cost and time of programming robotic systems. We would expect such robots to perform tasks that people do, tasks that a person can easily demonstrate.

Novel applications for robots such as cleaning, elderly assistance, house helping have became hot topics. These applications tend towards a society where robots will play a key role in our daily lives [13, 16]. From the engineering point of view, to success in these novel applications robots should display effective human-robot interaction, efficient coordination, adaptation, and learning; to mention few skills [3, 10]. Robots such COG, Kismet and ASIMO have demonstrated to be able to interact friendly and efficient with humans besides recognise grins and commands. 
However, these robots are still far from the level to help humans with their daily activities, mainly because learning new tasks is still a great challenge. It is one of the most complex abilities that humans have that involves many other skills. Current works in this area demonstrate the feasibility of early stages of robot's learning abilities. One learning methods that has received special attention is imitation. Imitation is the ability to learn novel actions by observing the performance of such actions. There are several challenges to equip a robot with the ability to imitate. Robots must be able to recognize their body parts and successfully map these parts with the body of the demonstrator of the action [2]. Finally robots should find an action that produces the same result as the observed action.

During the last decade we have seen several robots with the ability to imitate tasks that vary from writing letters, swing a racquet, and dancing for mention few examples. However, being able to reproduce a task does not mean to have the ability to apply this knowledge to solve a similar task. Learning with imitation deals with learning new abilities from imitation as well as to apply the new knowledge to new tasks [14]. This paper deals with learning by imitation focusing on actions with objects. The level of imitation pursued should focus on the reproduction of the goal, which means not just to copy the movements but to try to get the same results $[9,12]$.

The rest of the paper is organized as follows. The second section presents the background theory and current works on imitation. The next section discusses in details of the use of the environmental state to achieve imitation of the robot actions with objects. Experiment results are presented in the third section. Finally, the last section concludes the paper and outlines the future work.

\section{Background}

Humans are able to perform huge variety of actions as well as to identify which actions are feasible with their bodies and which are not. The information used by the body in order to carry out an action is derived from two sources [20]:

1. The body schema is the long-term representation between the spatial relations among body parts and the knowledge about the actions that the body can and cannot perform.
2. The body percept refers to a particular body position perceived in an instant. It is built by instantly merging information of the body schema with two sources: sensory (including visual) input, and proprioception. It could be considered as the awareness of a body's position at any given moment.

The combination of the information from the body percept with that from the body schema will help to identify and reproduce actions. For instance, by observing someone, one can easily determine how one would accomplish the same task by using one's own body. This means that it is possible to recognize the action that someone else is performing. This is possible since the body schema, a body representation with all its abilities and limitations, along with the representation of other objects can simulate any given action. The body schema provides the basis to understand similar bodies and perform the same actions [17]. This idea is essential in imitation. In order to imitate, it is first necessary identify the observed actions, and then be able to perform those actions. Body schema is able to support these abilities by two main functions:

- The forward models inside the body schema help to calculate the position of the body parts after an action has being performed.

- The inverse models loop-up for an action that satisfies a goal position from the current position of the body parts.

The functionality of these models has been referred with different terms in areas such control. Whereas, the forward model has been referred as controller or direct function, the inverse model has been known as predictor or inverse function. However, the terms forward and inverse models are well adapted by the research society on cognitive learning with robots.

It is believed that the interaction of these models allows humans to produce a mental performance of actions [15]. This mental rehearsal of actions helps us to improve actions, and adapting our bodies to new actions before to execute the actual action. In a similar way, these models interact when observing someone performing an action; we can image ourselves performing the same action. This process of imaging oneself performing the observed action is called Direct Imitation [11]. The idea that the forward and inverse model are used to these mental rehearsals finds support in the fact that the mental actions are constraints by only 
realistic physically movements with one's body [19]. The interaction between the body schema and the body percept permits us to understand the relationships between ourselves and other's bodies. Thus, in order to achieve a target perceived action a mental simulation is performed constraining the movements to those that are physically probable.

\section{Objects and actions}

Learning a new task is not just a trivial process for humans. We learn a task through a mechanism of learning and this learning process is supported communication and interaction. Eventually, after multiple observations and performances, we are able to perform the new task. Experiences and personal knowledge are used to refine the learned task. The purpose of the task helps us to adapt the actions to achieve a task when the conditions have changed.

Robots in a similar way must learn to perform new tasks and be able to adapt their actions to the dynamic environment. Adaptation of the actions required awareness of the goals to attain.

Extracting the purpose or goal from an action is not a trivial task. Humans require observing and performing an action perhaps more than once, thus, through a higher cognitive mechanism, humans are able to extraction the goal. The current limited capabilities of robots make the extract of a goal a complicated task. Nevertheless, there are features in the state of the robot and the environment that could be used as indicator whether a goal has been achieved or not. Robots could aim to monitor these values in the state to associate them with actions creating schemas that could in future be reproduced.

Let us simplify a goal of an action as a set of the different values in the environment that change from an original state A to a goal state B. Using the former definition, the goal of task can be seen as a collection of sub-goals that must be achieve with a particular order. Thus, a robot can learn to associate the goals of the actions in the task in a specific order, and thus the robot will be able to reproduce the task [7].

Representing a task by a set of goals provides great flexibility in the execution of the task. A robot could more than one action to achieve a goal, thus an action could be replace by another to achieve the same goals, when the original action fails. The previous idea is greatly accepted when the environment is different from the one where the task was learned [6]. In addition to this adaptability, the robots could take advantage of working with the goals rather with the actions, thus they could use actions that are more suitable to their body structure.

One important consideration is to establish the relation between the body of the agent performing the task and the objects involved in the task. The representation of our bodies is treated different from the representation of other objects, and the actions of our bodies on objects hold a dual representation. Actions have an abstract representation used for planning and its execution. Studies also suggested that body-motor part representation also helps to organize and to identify the actions [22]. This second representation is more related with to identify an action by observing someone performing it; this representation helps to find in one's repertory of action the suitable match for the observed action. Thanks to this representation is also possible to identify new actions that are not part of one's repertory.

Let us define an action as a transition or affordance from an initial state (namely pre-conditions) to produce a final state (namely post-conditions). According to this definition, an action would consist of three elements, a transition, an initial state, and a final state. Both the initial and the final states are state values, which represent the condition of the environment, the robot and the relation environment-robot. These values can be discrete, e.g. "State of the gripper" (Open or Close); or continuous, e.g. "Distance to the object" $(20 \mathrm{~cm})$

The pre-conditions are the state of the relation environment-agent before the transition can be executed. On the other hand, post-conditions are values that express the resulting state of the environmentrobot relation after the transition has been taken. The post-conditions can be interpreted as the embedded goals of the action [18]. An example of an action is, to perform the action grab on the object $\mathrm{A}$, the pre-conditions ask that the object $\mathrm{A}$ must be within a reachable distance, and that the gripper is not carrying another object. Once the action is executed, the post-conditions should be that the object $\mathrm{A}$ is within the fingers of the gripper. The condition of the environment can be modified by the robot's actions.

In general, the robot's choice of action at any given instant depends on the entire body percept sequence observed to date [21]. However, to analyse all the body percept in the robot history makes the selection of an 
action a tough job. To present a better approach to this problem, the actions can be selected based on the current percept state and the goal state that the robot has to achieve. Nevertheless, the body percept can be interpreted in two different ways according to whether the robot is observing to imitate or performing an action. When the robot is about to perform an action the body percept contains the relation of the environment-robot, and it is used as pre-conditions of the action about to perform. In contrast, during a demonstration, the body percept is used as the goal to be achieved.

The environment-robot state values of the body percept are calculated by using the current reading from the sensorial, visual, and proprioception data. For example, the value for Gripper State is obtained directly from the sensory input, checking if the gripper is either closed or open. Nevertheless, in some cases we not only use the current sensorial readings at time $t$, but also those of previous states of the percept sequence $\left[t-1, t-2, \ldots, t_{0}\right]$ as well. For example, in the case of the value of Approaching, the robot uses the previous states to check if the end-effector was far from an object, and now getting closer to an object.

The body schema, not only contains information about the physical parts of the body, but it also keeps the knowledge of which actions can be performed. This knowledge about feasible actions is used to recognise those actions when they are executed. Thus, the main two functions of the body schema regarding actions are stated as:
1. Forward model, it is the action being taken when the robot has an action to perform and the preconditions in the current body percept $(t)$ are met. Then the goal of the action is achieved.

2. Inverse model, this situation arises when the robot knows the goal that it has to achieve, which is contained in the body percept $(t+1)$. Besides, the robot knows its current body percept $(t)$, which is a pool of possible pre-conditions. Thus, the robot can look an action up in its repertoire, that action would need to satisfy the goal and its preconditions must be met.

The robot's body schema has been equipped with an initial set of actions known as primitive actions. The majority of these actions are atomic actions, which can be executed in one step. In contrast, a complex action is an action that contains at least two actions whether they are atomic or not. The robot is able to create new complex actions by employing the original set of primitive actions. The actions in a complex action are connected as a net by their pre-conditions and postconditions. This complex action can be seen as a plan to achieve a particular task.

All the primitive actions related to a particular object are collected in an object schema. The object schema is implemented as a finite state machine (FSM), in which the states are the pre-conditions and post-conditions; whereas the links between the states are the transition of all the actions (see Fig. 1). One advantage of the FSM

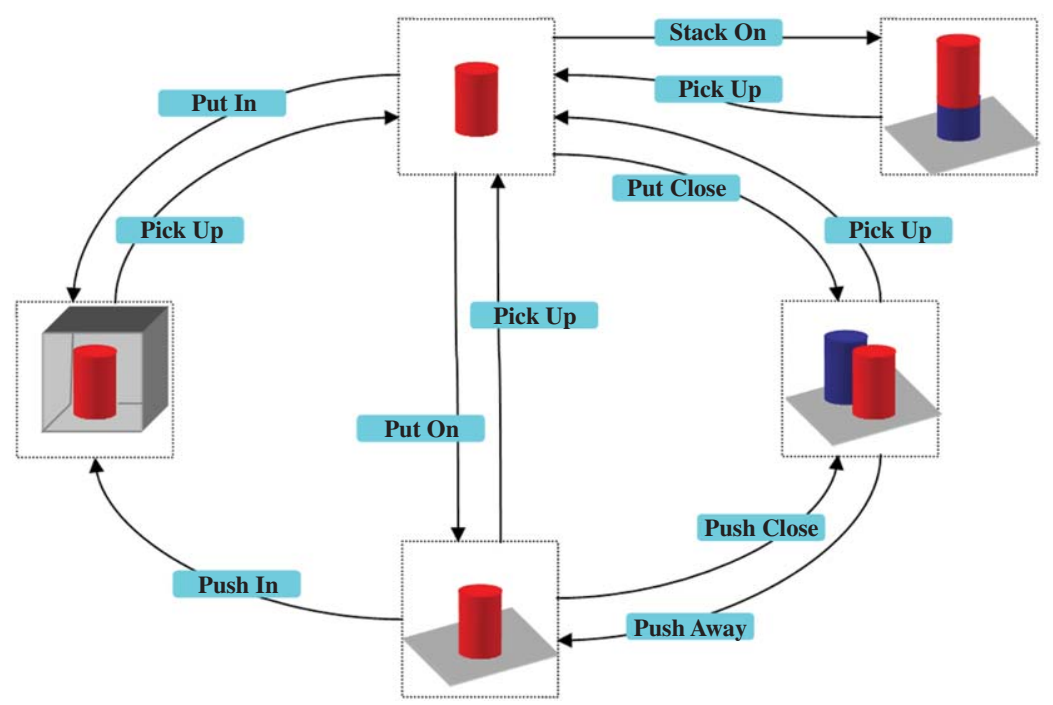

Fig. 1. An object schema representation for the possible set of primitive actions for the object cylinder. 
representation is that they tell us about the necessary sequence of actions from an internal state to a goal state for a particular object.

The object schema representation defines the apriori knowledge about a specific object. It only tells the imitator which actions can be performed and which action cannot be performed on the object. Furthermore, the forward and inverse models of the body schema exploit this representation. The object schema combines the dual object representation; the abstract representation that helps us to plan actions, and the body-motor representation that help us to identify an action when observed.

\section{Mechanism of imitation}

We have mention in previous sections that the object schema and the functions of the body schema only establish what the imitator can do with the object, but they do not tell the imitator how to achieve a task. The mechanism of imitation, described in this section, allows the imitator to learn the necessary actions to accomplish a task. The aim of the mechanism is to achieve the same sequence of goals as the ones observed from the demonstrator. The representation of a sequence of goals in the system is by a complex action, which contains a sequence of actions used to achieve those goals. Thus, the complex action defines a way to solve a task.

This mechanism works in two complementing ways depending on whether the robot is observing to imitate or performing an action. Table 1 shows the relation between the body schema and the body percept when observing and performing an action.

When the imitator is observing to imitate the body percept will contain the environment-demonstrator state, which is to simulate what the demonstrator experiences. A change in the state of the demonstrator by an action is registered as a new body percept represented as percept $(t+1)$. Therefore, the robot will interpret this change as a new goal, i.e. the difference between its current body percept $(t)$ and the new body percept $(t+1)$.

$$
\text { Goal }=\text { BodyPercept }(t+1)-\operatorname{BodyPercept}(t)
$$

The body schema is able to employ the inverse model to search for an action that satisfies the criteria of the new goal. The inverse model uses the body percept $(t)$
Table 1

The difference between the body schema and body percept when observing and performing an action

\begin{tabular}{|c|c|c|}
\hline Feature & Observing & Performing \\
\hline \multicolumn{3}{|l|}{ Body percept } \\
\hline $\begin{array}{l}\text { Type of } \\
\text { relation }\end{array}$ & $\begin{array}{l}\text { Environment- } \\
\text { demonstrator }\end{array}$ & $\begin{array}{l}\text { Environment- } \\
\text { imitator }\end{array}$ \\
\hline Percept $(t)$ & $\begin{array}{l}\text { Pre-condition } \\
\quad \text { (Known) }\end{array}$ & $\begin{array}{l}\text { Pre-condition } \\
\quad \text { (Known) }\end{array}$ \\
\hline Percept $(t+1)$ & $\begin{array}{c}\text { Post-condition } \\
\text { (Known) }\end{array}$ & $\begin{array}{c}\text { Post-condition } \\
\text { (Unknown) }\end{array}$ \\
\hline \multicolumn{3}{|l|}{ Body schema } \\
\hline Action & Unknown & Known \\
\hline Function & Inverse model & Forward model \\
\hline
\end{tabular}

as pre-conditions and the body percept $(t+1)$ as postconditions. A relation of the possible relevant objects for the action is extracted from the body percept $(t)$ and $(t+1)$. This relation of the possible relevant objects leads the inverse model to search into specific object schema, where pre-conditions and post-conditions narrow the search to an action.

The selected action is then passed to the forward model to predict the state after the execution of the action. These values of the predicted state are compared with the ones in the body percept $(t+1)$; thus, one of the following situations may occur:

- If the predicted state is the goal defined in Equation 1 , then the action is added into the complex action.

- If the predicted state contains both the goal and extra values that are not part of the body percept $(t+1)$; then these values are added to the body percept $(t+1)$ and the action is added into the complex action.

- If the predicted state does not satisfy the goal, then the action is discarded and other relation of objects is used to find another action.

This learning process is depicted in Fig. 2. The imitator can either, perform an action and send its signals to the effectors, or it can inhibit the physical motion thereby producing a mental simulation of the action.

Executing a learnt action involves a different relation of the body schema and the body percept. The body percept will now contain environment-imitator state, i.e. the body percept $(t)$ is the current relation of environment and the imitator. The complex action to be executed is then decomposed into its primitive actions. Each action $A_{i}$ is executed by the following these steps: 


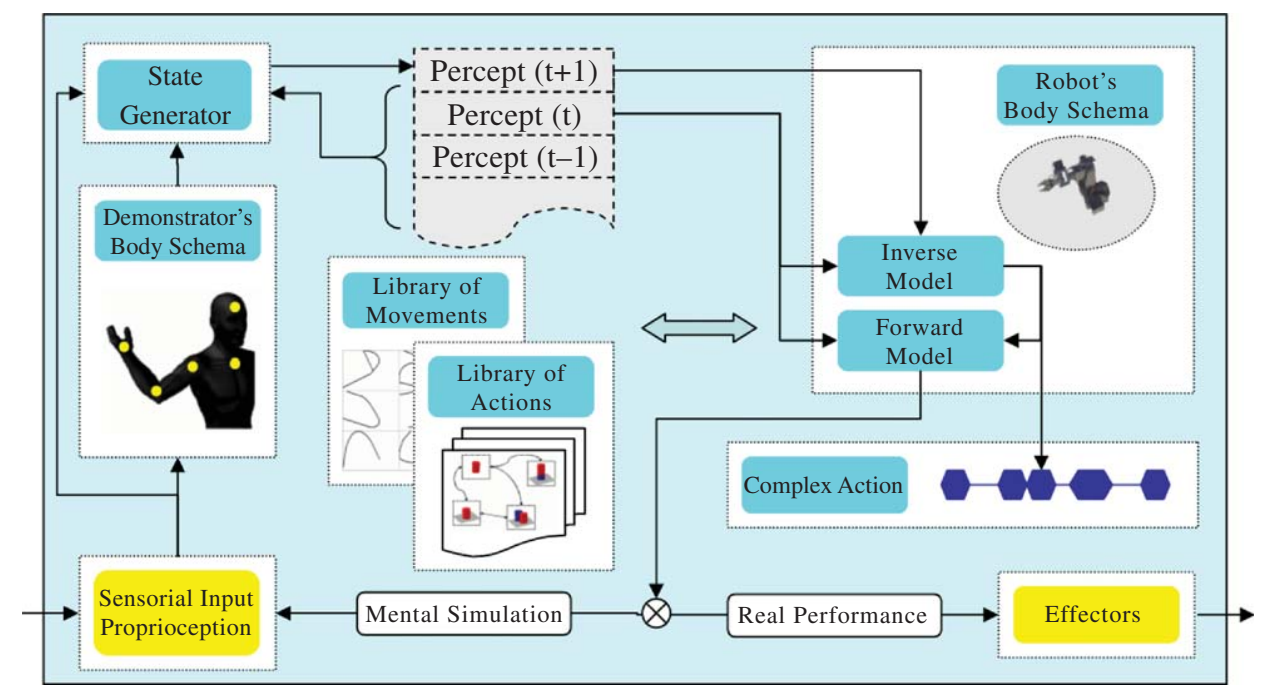

Fig. 2. The interaction of the body percept and the body schema, when the robot system is learning. The output from an action can be inhibited to the effectors, which will produce a mental rehearsal of the action.

1. It is checked whether the pre-conditions of action $A_{i}$ are part of the current body percept $(t)$. If they are part of the body percept $(t)$ then step 2 is taken. But if the pre-conditions are not met then the inverse model is called to search for an action that once executed satisfies the pre-conditions of $A_{i}$. In other words, the body schema will search for an action $A_{i-1}$ to be executed before $A_{i}$, and once $A_{i-1}$ is executed it will produce the required state for $A_{i}$ being executed. Therefore, the body percept $(t)$ becomes the pre-conditions for the inverse model, and the pre-conditions of $A_{i}$ become the post-conditions of the inverse model. If an action is found by the inverse model, which satisfies the pre-conditions of the action to be executed, then this new action must be executed first, and it is sent to step 2. However, if no action is found then the system reports the error and stops the execution of the complex action.

2. The action $A_{i}$ is executed by the forward model of the body schema, which produces the expected environment-robot state. Hence, the motor control receives both the motor commands for actuators and the expected environment-robot state. The sensors keep registering the values until either these values fulfill the expected environment-robot state or the watchdog-time is up. If the post-conditions of $A_{i}$ are not reached, the system could try again the same motor commands or other that produce the same effect, when these exist. If these motor commands fail, the current environment-robot state is reflected into a new body percept $(t+1)$ and the inverse model searches for an alternative action. This time the inverse model uses the new body percept $(t+1)$ as pre-conditions and the post-conditions of $A_{i}$ as post-conditions. If an action is found this must be executed and it is sent to step 2. When no action is found the system reports the error and stops.

3. When the action $A_{i}$ is performed the environment-robot state values perceived in sensors are reflected into a new body percept $(t+1)$. If there are more actions then the next action $A_{i+1}$ is sent to step 1; until the complex action is finished.

The execution of a primitive action requires searching for the primitive action in the robot's library of actions. On the other hand, to execute a complex action the forward model has to make a recursive call for each single action that compounds the complex action, until it finds the primitive actions that make the complex actions. The forward model also monitors that the effects of the executed action are obtained, by checking them in the body percept $(t+1)$.

One aim of personal robots is to help their owners in their daily tasks. These personal robots would require learning new tasks in order to be helpful for the specific needs of their owners. Consider the following situation: The robot is observing from its teenager owner how 
to obtain a can of soda from the fridge. The teenager approaches the fridge. He scratches his head and then opens the fridge. He grasps the can of soda with his left hand and then passes the soda to his right hand while he rubs his left hand against his leg to ease the cold sensation. The demonstrator closes the fridge door with his hips and winks an eye to the robot.

The question here how does the robot decide what action are relevant and what action are not from this performance. Any irrelevant actions to achieve a goal can be particularly difficult to identify from this observed performance. A way to solve this situation is to observe several performances of this task until the imitator is able to segregate the relevant actions that lead to the goal of obtaining the can of soda from the fridge.

However, observing several performances of actions bring another question, how to recognize when an action has been previously observed. It is important to recognise whether an action has been observed before or not. Finally, if the action has been observed before it is necessary to find a way to merge similar performances of the same action into a single functional representation, that then it could be used for its performance.

\subsection{Action representation}

The environment-robot relation is represented by abstract states in the body percept. This state record the condition of the environment at a given instant, much like a snapshot, and we represented as body percept $(t)$. The robot's percept sequence, from time 0 to the current time, can be thought of as a history of what the robot has perceived during its lifespan [21].

The condition of the environment can be modified by the robot's actions. In general, the robot's choice of action at any given instant depends on the entire percept sequence observed to date. However, to analyse all the body percepts in the sequence makes the selection of an action a tough job. To present a better approach to this problem, the actions can be selected based on the current percept state and the goal state that the robot has to achieve. Thus, the representation of an action has the following characteristics:

- Pre-conditions are state values that must be met before the execution of the action. These state values are contained in the current robot's body percept $(t)$.
- Post-conditions are state values obtained after the execution of the action. These states comprise the next robot's body percept $(t+1)$. The action's post-conditions are interpreted as the embedded goals of the action [18].

The action is a piece of code responsible for sending high-level requests for commands or commands sequences to the robot's actuators. The robot's body schema has been equipped with a set of primitive actions, which are the basic actions that the robot can perform. The robot is able to build complex actions by using these primitive actions. The representation of a complex action is a net of actions connected by preconditions and post-conditions. The net corresponds to a plan to achieve a particular task. The actions in the net could be either primitive actions or complex actions. The set of primitive actions in the library would essentially outline by the robot-environment states that the robot is able to recognize.

The robot-environment states' values of the body percept are calculated in some cases by using the current reading from the sensorial, visual, and proprioception data. For example, the value for Gripper State is obtained directly from the sensory input, checking if the gripper is either closed or open. Nevertheless, in some cases we not only use the current sensorial readings at time $t$, but also those of previous states of the percept sequence $[t-1, t-2, \ldots, 0]$ as well. For example, in the case of the value of Approaching, the robot uses the previous states to check if the endeffector was far from an object, and if it is getting closer to that object.

\section{Identification of an action}

As mentioned in the previous Section, actions are group into a library of actions. Inside this library action could be group in atomic or primitive actions, which are actions that can be executed in one single step; and complex actions, which are actions that consist of a net of sub-actions interconnected by their pre-conditions and post-conditions. The identification process helps to determine whether an observed action is already part of the library or it is a novel action. Let a library's complex action consist of a sequence of sub-actions:

$$
A=\left[a_{0}, \ldots, a_{i}, \ldots, a_{M}\right]
$$


where $a_{i}$ is the sub-action in the position $i$ of the complex action $A$. Assume that in every unit of time an action is performed, we consider the time discrete since the environment states are not continuous on time. Let a new complex action consist of the following sequence:

$$
X=\left[x_{0}, \ldots, x_{i}, \ldots, x_{N}\right]
$$

where $x_{i}$ is the sub-actions added in the position $i$. In the time $i$ of the demonstration, the body schema of the imitator employs the inverse model to pick up a candidate action, which is validated by the forward model. Once an action $x$ is confirmed by the forward model, then this action $x$ is added to a complex action $X$ that represents the current demonstration.

The identification of $X$ as a library template during the demonstration is achieved by the computation of the confidence value of the complete action. The identification of an action when it is observed may be a heavy calculation especially in a system with many actions in the library. The confidence for a library action $A$ is computed by

$$
\alpha A_{i}=\alpha A_{i-1}+\delta(i, j) * \sigma(i, j)
$$

where $\alpha A_{i}$ is the confidence value of the complex action $A$ at the time $i$, and $\delta(i, j)$ is a proportional value defined as

$$
\delta(i, j)=\frac{j}{M} * i
$$

and $\sigma(i, j)$ is the reward value defined as

$$
\sigma(i, j)=\left\{\begin{array}{lr}
a_{j}=x_{i}, & 1 \\
a_{j} \neq x_{i}, & -1
\end{array}\right.
$$

The $M$ value is the number of actions in the library complex action $A$. The index $j$ corresponds to the number of common actions between of the sequence $A$ and the demonstration $X$ at the time $i$. The discrete time $i$ increases when a new sub-action $x$ is registered from the demonstration. In contrast the index $j$ is only increased when the sub-action $a_{j}$ equals to the sub-action $x_{i}$ of the demonstrator.

When an action $x$ in time $i$ is validated by the forward model and added to the complex action $X$, then the other complex actions in the library start competing and their confidence values are rewarded correspondingly with Equation 4. Therefore, after several actions the confidence value of each library action will grow up or down accordingly if the sequence of actions of the demonstrator is close to the actions in the library [4].

Nevertheless, ending the demonstration being the highest confident among the complex actions of the library does not necessarily mean that the demonstrated action has been identified. It only means that among all the other complex actions this is the one closest in sequence with the observed demonstration. It is also necessary to evaluate the percentage of actions that were identified in the complex action as well as the percentage of shared actions from the demonstration. These two values of the most confident action could then be assessed to decide whether the demonstration is the same as the complex action in the library or not.

\subsection{Objects and actions}

The body schema not only contains information about the physical parts of the body, but it also keeps the knowledge of which actions can be performed. This knowledge about feasible actions is supposed to be used to recognize those actions when they are executed. Therefore, we can distinguish two main functions of the body schema regarding actions:

- Direct action is when the robot has an action to perform and the pre-conditions, in the current body percept $(t)$, are met. Then the action is taken and the goal of the action is achieved.

- Inverse action, this situation arises when the robot knows the goal that it has to achieve, which is contained in the body percept $(t+1)$. Besides, the robot knows its current body percept $(t)$, which is a pool of possible preconditions. Thus, the robot can look an action up in its repertoire. That action would need to satisfy the goal and its preconditions must be met.

These two functions of the body schema are important to achieve imitation. The inverse action is used to identify the action performed by the demonstrator; and the direct action predicts the next body percept. The imitator could either perform the action by sending commands to the effectors, or it can inhibit the physical motion and produce a mental simulation of the action.

The relation between the body schema and the body percept changes depending on whether the robot is learning or performing an action. When the robot is learning, the body percept contains the effects of the 


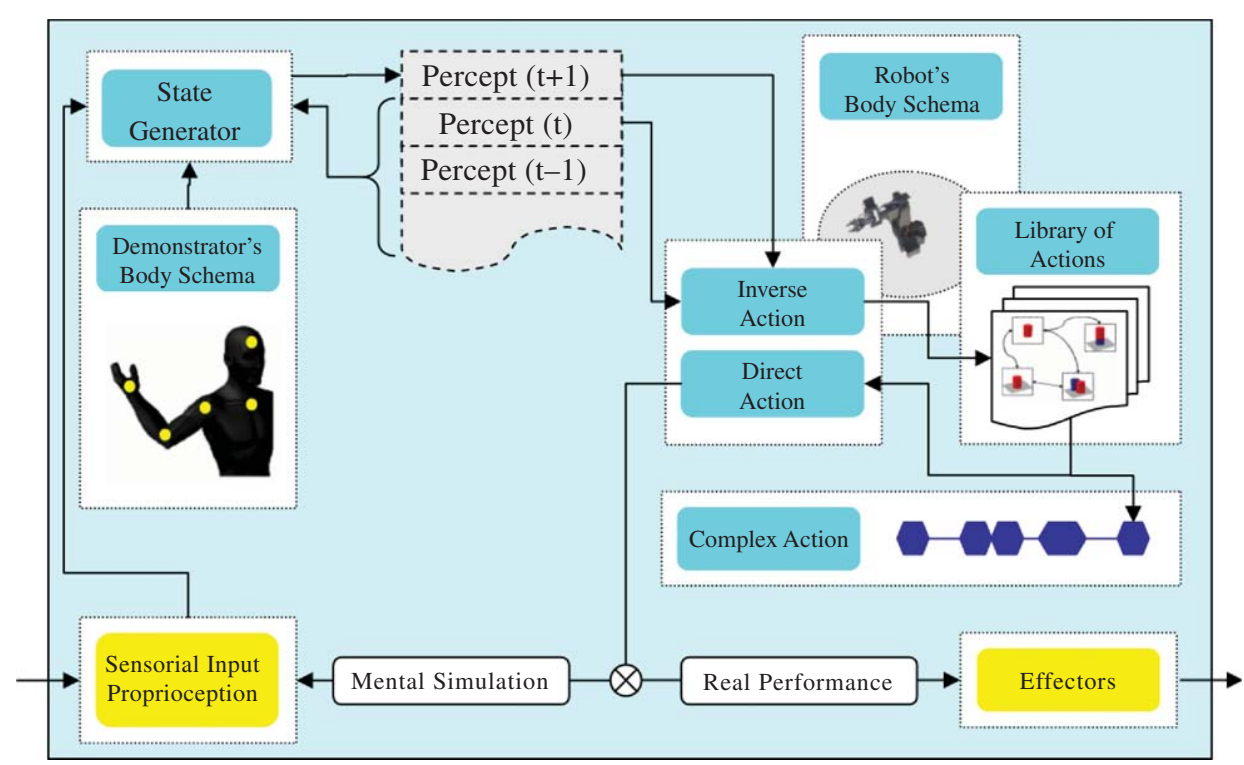

Fig. 3. The interaction of the body percept and the body schema, when the robot system is learning. the output from an action can be inhibited to the effectors, which will produce a mental rehearsal of the action.

demonstrator's actions upon the environment. This is because the system is simulating the states of the demonstrator in order to identify the actions. When a new action is performed a new body percept $(t+1)$ is created. This would act as the goal that the robot would need to reach from its current body percept $(t)$. The inverse action function of the body schema is then able to select an action that satisfies the criteria. In addition, the direct action function can predict the states generated after the execution of the action, which complement the states in the body percept $(t+1)$. This learning process is depicted in Fig. 3.

When the robot is executing an action, the body percept $(t)$ includes all the states of the robot and the state of the relation of environment-robot. The action that will be executed is then given to body schema, or more specifically to the direct action function. Here, it is first checked that the pre-conditions of the action are satisfied by the states in the current body percept $(t)$. The action will not be executed if the preconditions are not met. Once the pre-conditions are met, the action is decomposed into the sub-actions that are part of the net of actions. A sub-action is taken when its preconditions are satisfied. The execution of a primitive action requires looking for the action into the library. On the other hand, to execute a complex action the direct action function has to make a recursive call for each single action that compounds its net, until it finds the primitive actions. The direct action function also monitors that the effects of the executed action are obtained, by checking them in the body percept $(t+1)$.

\section{Experimental results}

In the previous sections the proposed imitation for mechanism has been presented. This section describes our experiments to investigate the abilities of the presented approach. The task selected for the experiments is the pick a ball and drop it into a box. The experiment was performed several times by different demonstrators. Each performance was observed by a Microsoft Kinect camera. The relevant objects in the environment were marker in different colours. In addition, during few performances the demonstrators were asked to do more the minimum actions required to complete the task, and in other cases to not complete the task. This pool of performances serves to prove the different features of the system.

The robot used in this experiment is NANSRY, this robot was built at the Advanced Robotics and Intelligent Control Centre (ARICC) at the Singapore Polytechnic. The robot is a humanoid torso with two degrees-of-freedom in the waist, two in the neck, two arms with five degrees-of-freedom each arm, and a hand with three motors that control the thumb, the 
index finger, and the three remaining fingers. The head of the robot is a Microsoft Kinect Camera. The robot has two speakers in the chest. In the base there are the board and PC104 that control the robot. The robot could be accessed via Ethernet or WIFI, and tasks can be running autonomously. The software that controls the robot is the same software architecture used in the RoboErectus Humanoid Robots that compete in the RoboCup Humanoid League [5]. In addition the OpenNI is used to obtain the depth information from the Kinect.

In the set-up the robot is facing the human, while observing the human demonstrator actions; and finally trying to reproduce the observed actions. The experiments were conducted in two phases for all the cases:

- Learning phase, in here the robot was observing the demonstrator's actions, while identifying the actions and recording them to be executed later.

- Execution phase, here the robot was set in an environment, which could be the observed one or similar and it must perform the actions learnt from the previous phase.

The relevant objects in the environment were marked with different colours, to simplify the extraction of significant information during the performance. The less cluttered background permits the robot to focus only on the relevant features. The joint information of the demonstrators is extracted by the OpenNI library; however, instead to try to map the human joints directly to the robot the system learn the relationship of the human joints to the robot joints, by understanding the differences and similitudes between systems. The understanding of the differences and similitudes is crucial is we would like to teach robots by demonstrating. The human and the robot have a different body structure. In out particular case the robot have only five degrees-of-freedom, but some of these degreesof-freedom have a wider range of mobility compare to those in the same position as the human. Besides the length of the links in the arm and the other parts of the body would not match between both systems, so it is necessary to scale the observed position of the joints while preserving the relation between the body and the relevant objects in the environment.

For these experiments the robot was endowed with a set of primitive actions. This set of primitive actions is mainly defined by the environmental states that the robot is able to recognize. The body percept is structured by both the position of the demonstrator's
Table 2

State values for the actions used in the pick and drop task

\begin{tabular}{cllllll}
\hline \multicolumn{6}{c}{ Action } & \multicolumn{5}{c}{ State value } \\
\hline \multicolumn{1}{c}{ Obj } & ObjMov GripObj ArmMov GripState \\
\hline Search & Precond & - & - & - & - & - \\
(obj) & Postcond & $\checkmark$ & - & - & - & - \\
Approach & Precond & $\checkmark$ & - & Far & - & - \\
(obj) & Postcond & $\checkmark$ & - & Close & - & - \\
Reach & Precond & $\checkmark$ & - & Close & - & - \\
(obj) & Postcond & $\checkmark$ & - & Touch & - & Open \\
Grab & Precond & $\checkmark$ & - & Touch & - & Open \\
(obj) & Postcond & $\checkmark$ & $\mathbf{X}$ & Touch & $\mathbf{X}$ & Close \\
Carry & Precond & $\checkmark$ & - & Touch & - & Close \\
(obj, path) & Postcond & $\checkmark$ & $\mathbf{X}$ & Touch & $\mathbf{X}$ & Close \\
\hline
\end{tabular}

end-effector and the states that represent the relation environment-robot. The state generator contains rules defining the behaviour of the relation robot-objects in the environment. For example, for the action (Carrying) an object the object must be in the Hand, and moving at the constant motion with the Hand.

In Fig. 6, the robot is performing the actions shown by the demonstrator of pick and drop. The description of the actions performed by the robot and theirs associated pre-conditions and post-conditions appear in the Table 2. First, it looks for the object, once this has been found (Search), the robot arm approaches to it (Approach). When the robot hand is close enough to reach the object, it stops (Reach) and finally it grabs the object (Grab).

In all the previous actions there is only one parameter involved, the object that the robot is handling. However, for the next action, (Carry), the robot requires two parameters: the object and the path to be follow by the arm. The path shown by the demonstrator when carrying the object cannot be used directly here from many reasons. Let's consider that the position of the ball and the box observed by the robot differs from the current setup where the robot is performing, if so the ball could be dropped outside the box. Thus the position of the ball and the box should be used as starting and ending points in the path. It is important to consider that the robot did not learn the action by observing it from one single demonstration and also it could be that many demonstrators and setups were involved to teach the robot this action. With this assumption in mind, the robot's imitation mechanism is using, as mentioned previously, the imitation of the goal, where the goal of this action is to carry the object above the box to then drop the ball. This means that the path follow by the demonstrators will have not a great impact in what the robot need to learn. 


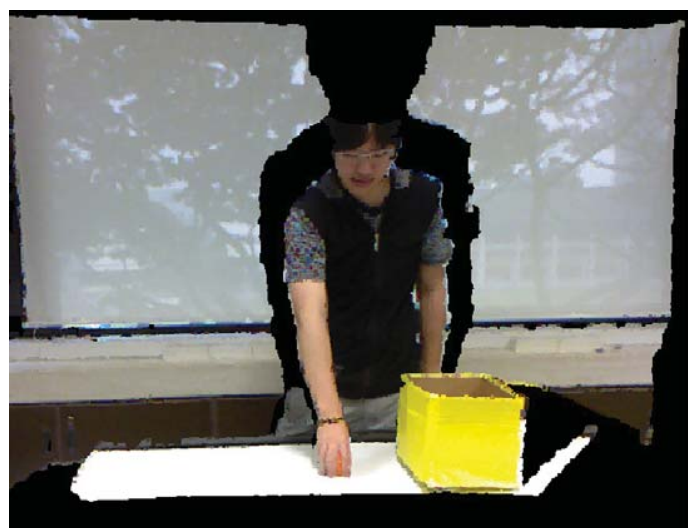

(a)

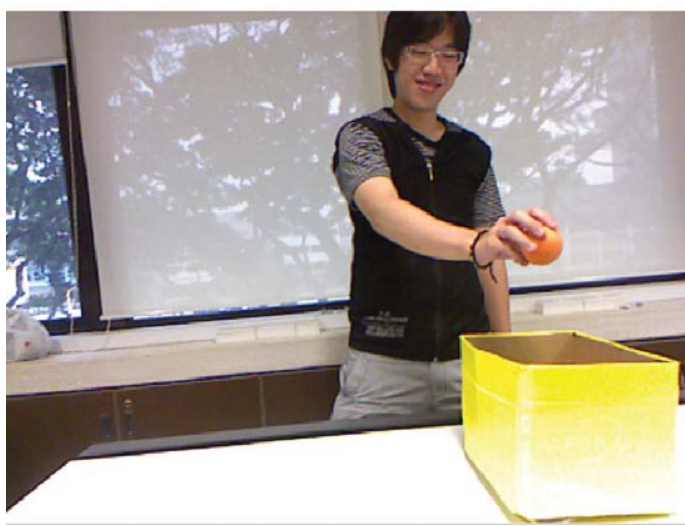

(c)

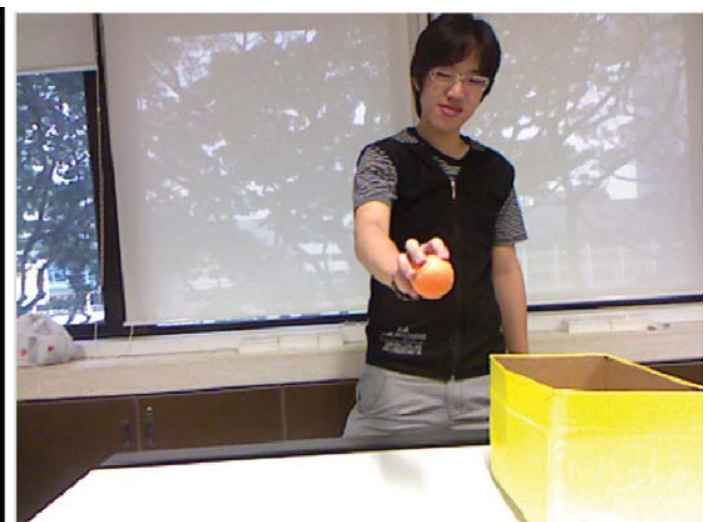

(b)

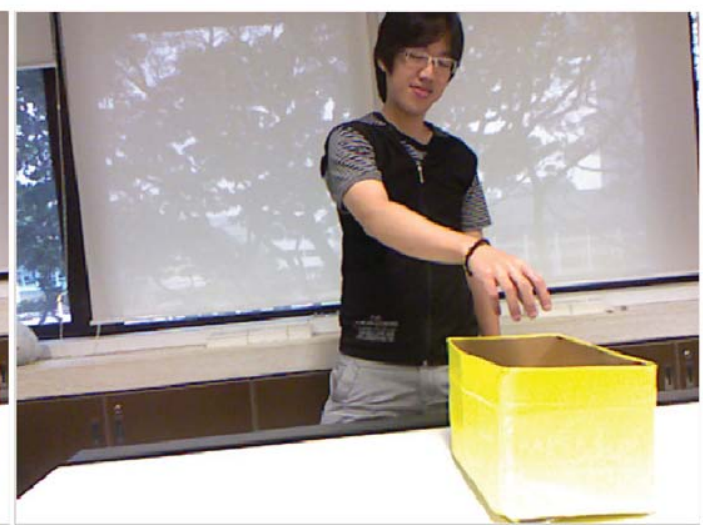

(d)

Fig. 4. One of the demonstrators performing the Pick and Drop task. The robot is placed in front of the demonstrator observing the task.

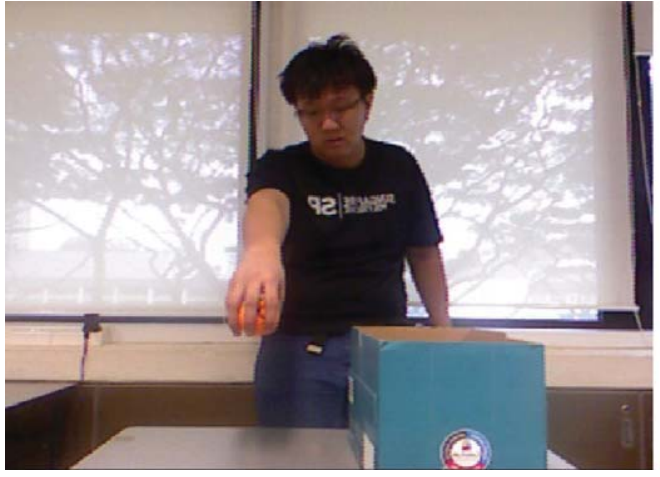

(a)

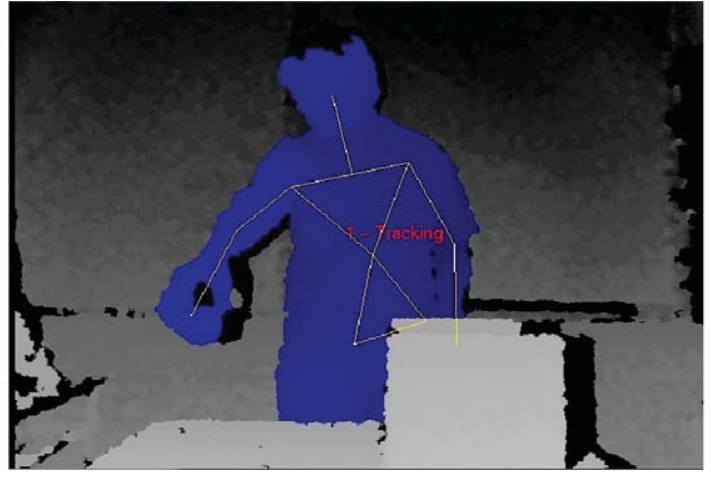

(b)

Fig. 5. Another demonstrator and its body information extracted with the OpenNI, the information of this body is merge with the colour objects to identify the actions performed with these objects, and the relation of the demonstrator body parts with the objects.

The robot is able to learn a path which is obtained based on all the observations of the performance of the action. Our previous work in [4] describes the cluster- ing method employed to obtain a representation path from many observed paths from different performers and setups. However, in here this representation 


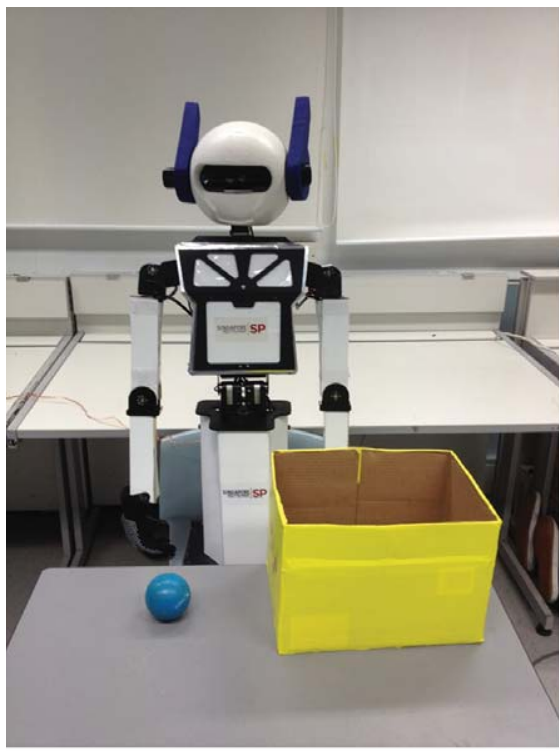

(a)

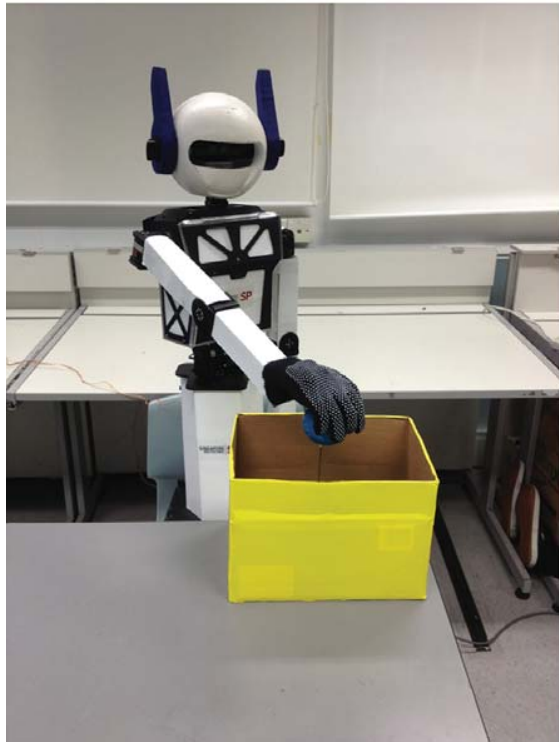

(c)

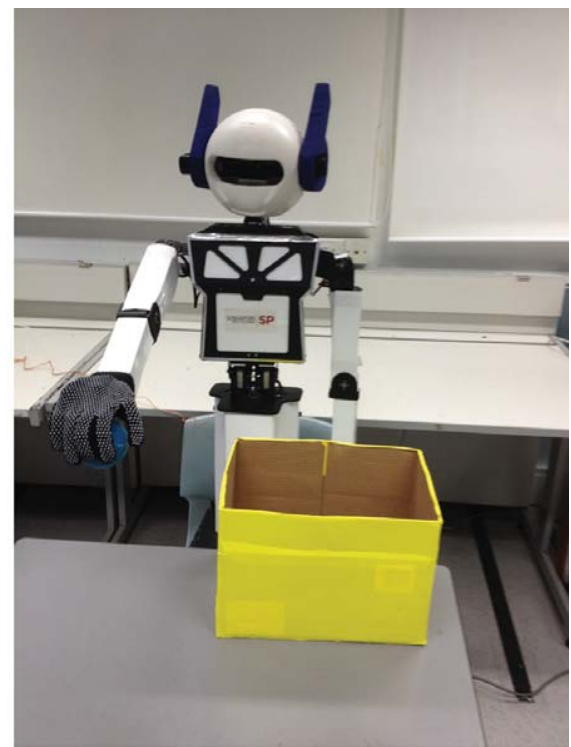

(b)

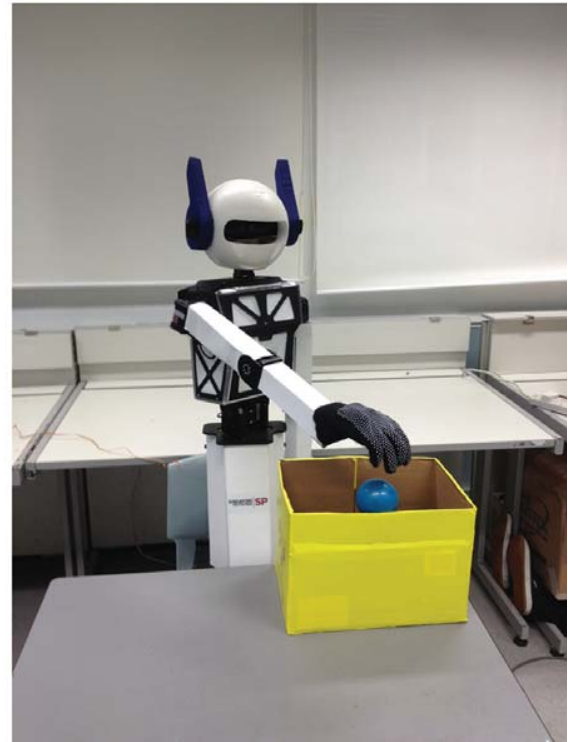

(d)

Fig. 6. The robot performance of the Pick and Drop task. See Fig. 4 to compare this with the demonstrators performance.

is adjusted by using interpolation to fit the position of the ball and the box.

A second experiment was conducted with RoboErectus $\mathrm{Jr}$ humanoid robot. This robot is a $60 \mathrm{~cm}$ tall robot mainly designed to play soccer in the Robocup Humanoid League (Acosta-Calderon et al. 2010). In addition the OpenNI is used to obtain the robot was modified to be able to grab objects with its right hand, but it only has two degrees-of-freedom in the arm plus the gripper. With this experiment we want to demonstrate that the difference of body do not affect outcome of the imitation when the goal and focus of the imitation is based on the objects and the actions.

For the learning stage, it was used the same setup as the previous experiment, however, since this robot 


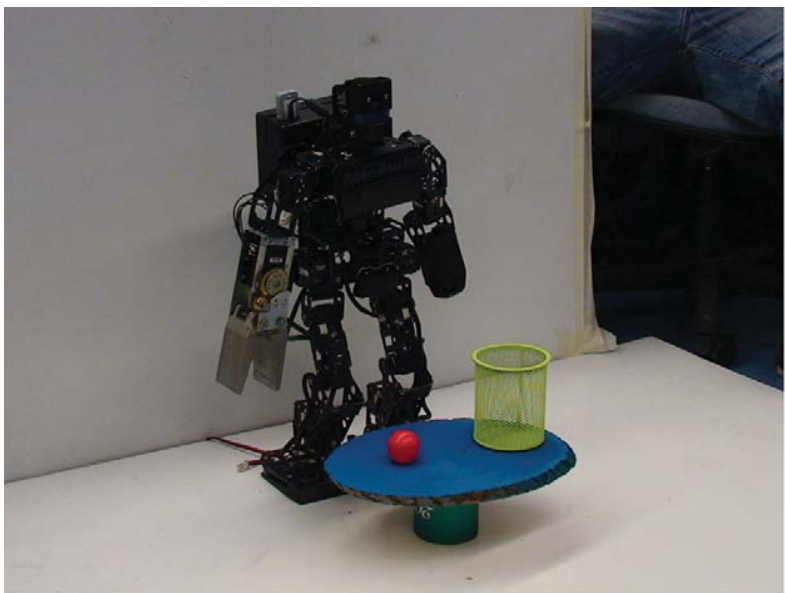

(a)

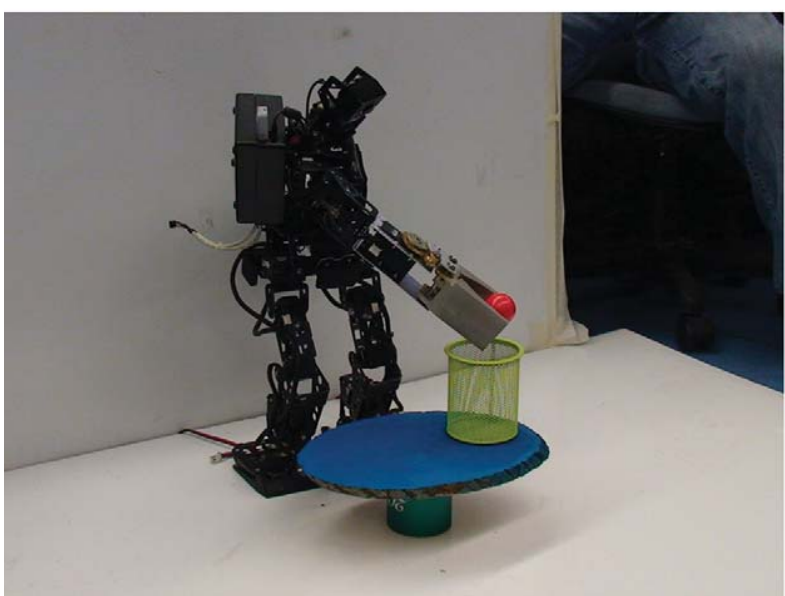

(c)

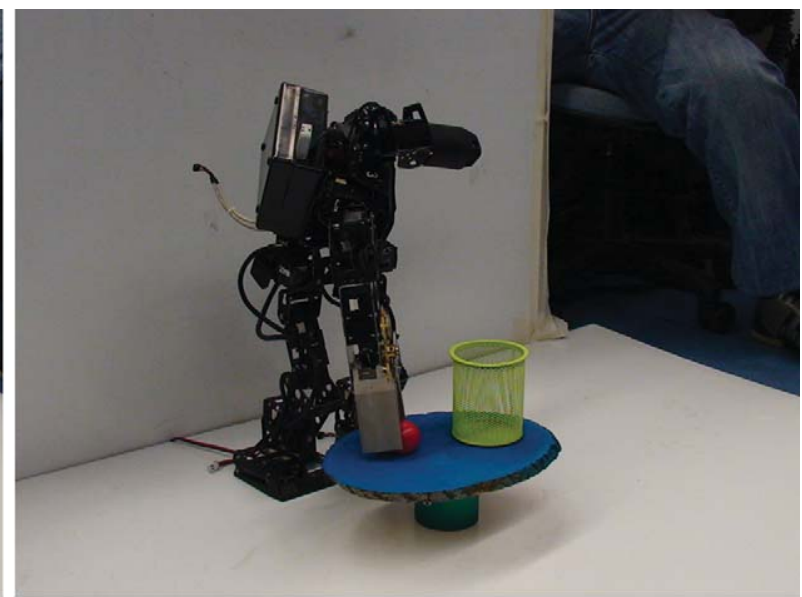

(b)

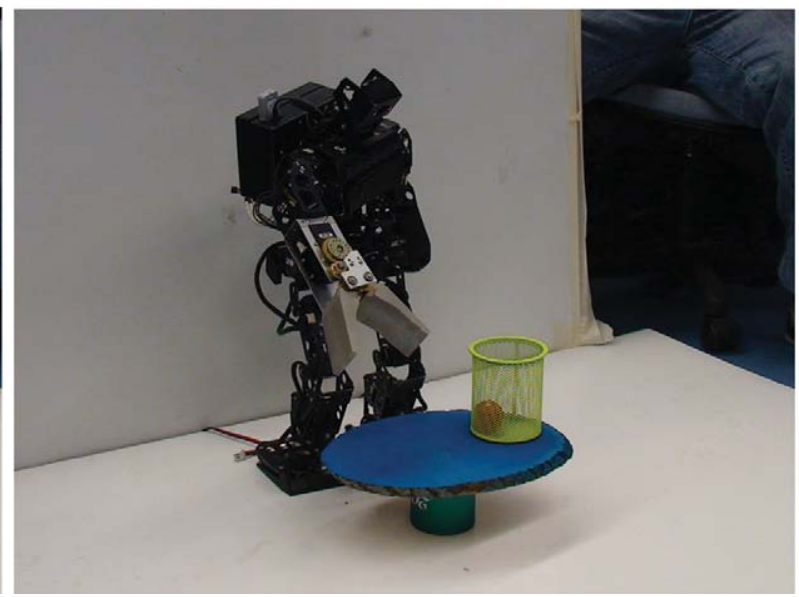

(d)

Fig. 7. The second experiment shows the performance with a different hardware. RoboErectus Jr a humanoid robot was used for the performance.

is only equipped with a webcam, a Kinect was placed in front of the demonstrator, the Kinect was connected to a PC that was responsible for extracting the relevant information of the performance identifying and connecting the actions; this is mainly because the processing power of the embedded system of the robot is limited, thus only motor command and sensor readings were transmitted and received from and to the robot.

For the executing stage, we indicate the relevant object for the imitation with colours that the robot is programmed to identify, so that the robot could use its camera for the performance. Figure 7 shows the performance of the robot, when the robot execute the approaching, reaching and grabbing, it is important to highlight the difference between this performance and the one of NANSRY in Fig. 6. NANSRY performance is close to the demonstrator because its body structure of the arm is similar. In addition, the approaching of NANSRY is also based on a similar path to the demonstrator, this is possible due to its control system for the arms, were paths can be defined to be followed. In contrast, RoboErectus Jr arm is limited to few motions, and its approaching, reaching and grabbing are programmed to always be executed in the same way, so for the robot it does not matter the path that the demonstrator shown with his arm. However, even with these differences in the system both robots are able to perform the same task.

One final remark, the relevant objects in the environment were indicated with colour but more importantly 
with the type of object. The colour is important to ease the identification for the robot, since object identification and recognition is not our priority here. But the type of object will provide more information to learn and execute the task, this is because the appropriate object schemas can be used with the objects now, this means that if the ball and the box switch places, then the robot would be able to perform the same task not only based on the position of the objects. This is why it is possible to change the box for a bucket in the performance of RoboErectus Jr.

\section{Conclusions}

Imitation offers important advantages as a learning mechanism for this reason imitation is seen as a real option to fill the gap to build social robots. Imitation is a tool that robots could use to acquire new knowledge, which could be applied to a variety of tasks and not only as a mechanism to solve a specific task.

This paper described our approach based on the body schema (which is in humans, the body representation and contains the physical limitation as well as the feasible action that the body can perform) and the body percept (which is the position of the body parts at a given instant, in addition it keeps the relation of the body with the environment). The body schema and the body percept give us an insight into how other people perform certain actions. Since the knowledge of feasible actions and physical constraints is implicit in the body schema, it is possible to do a mental rehearsal of other peoples' actions and gather the results of those actions at particular body percepts for the body schema. These two key components play a crucial role to achieve imitation. This paper describes how these components interact to achieve imitation of actions on objects, involving the identification of an action by using the body schema and using the body percept as the target goal.

We have also described our experiments with two different robots as imitators, imitating the movements of a human demonstrator. The experiments show the feasibility of the proposed approach for imitation of actions on objects. Even with different bodies it is possible to perform the same task, the reason for these is the goal or focus of the imitation is based on the actions and the object. In previous works, it was demonstrated that different embodiment affect the performance of tasks when the goal or focus is to imitate the exact movements of the demonstrator, e.g. when learning a dance.

\section{References}

[1] C.A. Acosta Calderon and H. Hu, Robotic societies: Elements of learning by imitation. In: Proceeding of the 21st IASTED Int Conf on Applied Informatics, Innsbruck, Austria, (2003), 315-320.

[2] C.A. Acosta Calderon and H. Hu, Imitation towards service robotics, In: Proceedings of Int Conf on Intelligent Robots and Systems IROS, Sendai, Japan, 2004.

[3] C.A. Acosta Calderon and H. Hu, Robot imitation: A matter of body representation,' In: Proceedings of Int Symposium on Robotics and Automation ISRA, Queretaro, Mexico, (2004), 137-144.

[4] C.A. Acosta Calderon and H. Hu, Robot imitation: Body schema and body percept, Journal Applied Bionics and Biomechanics 2(3-4) (2005), 131-148.

[5] C.A. Acosta Calderon, R.E. Mohan, L. Hu, C. Zhou, and H. $\mathrm{Hu}$, Generating Human-like Soccer Primitives from Human Data, International Journal of Robotics and Autonomous Systems 57(8) (2009), 860-869.

[6] A. Alissandrakis, C.L. Nehaniv, K. Dautenhahn and J. Saunders, Achieving corresponding effects on multiple robotic platforms: Imitating in context using different effect metrics,' In: Proceedings of Third International Symposium on Imitation in Animals and Artifacts at AISB05 (2005), 10-19.

[7] A. Alissandrakis, C.L. Nehaniv, K. Dautenhahn and J. Saunders, An approach for programming robots by demonstration: Generalization across different initial configurations of manipulated objects, In: Proceedings of IEEE Computational Intelligence in Robotics and Automation CIRA2005 (2005) 61-66.

[8] P. Bakker and Y. Kuniyoshi, Robot see robot do: An overview of robot imitation, In: Proceedings of AISB96 Workshop on Learning in Robots and Animals, Brighton, England, (1996), 3-11.

[9] A. Billard, Y. Epars, S. Calinon, G. Cheng and S. Schaal, Discovering optimal imitation strategies, robotics and autonomous systems, special issue, Robot Learning from Demonstration 47(2-3) (2004), 69-77.

[10] C. Breazeal, D. Buchsbaum, J. Gray, D. Gatenby and B. Blumberg, Learning From and About Others: Towards Using Imitation to Bootstrap the Social Understanding of Others by Robots, to appear in Artificial Life, 2004.

[11] Y. Demiris and M. Johnson, Distributed, predictive perception of actions: A biologically inspired robotics architecture for imitation and learning, Connection Science 15(4) (2003), 231-243.

[12] K. Dautenhahn and C.L. Nehaniv, The Agent-Based Perspective on Imitation, Imitation in Animals and Artefacts, C.L. Nehaniv and K. Dautenhahn, Eds., The MIT Press, Cambridge, MA, 2002.

[13] T. Fong, I. Nourbakhsh and K. Dautenhahn, A survey of socially interactiver robots, Robotics and Autonomous Systems 42 (2003), 143-166.

[14] M. Johnson and Y. Demiris, Perceptual perspective taking and action recognition, In: Proceedings of International Journal of Advanced Robotic Systems 2(4) (2005), 301-308. 
[15] A. Goldman, Desire, Intention, and the Simulation Theory, Intention and Intentionality, F. Malle, L. Moses and D. Baldwin, Eds., MIT Press, Cambridge, MA, 2001, 207-224.

[16] J. Liu and J. Wu, Multi-Agent Robotic Systems, CRC Press, London, 2001.

[17] A.N. Meltzoff and M.K. Moore, Imitation, memory, and representation of persons, Infant Behaviour and Development 25 (2002), 39-61.

[18] M.N. Nicolescu and M.J. Mataric, Experience-based representation construction: Learning from human and robot teachers, In: Proceedings of IEEE/RSJ Int Conf on Intelligent Robots and Systems, Hawaii, USA, (2001), 740-745.

[19] R.P.N. Rao, A.P. Shon and A.N. Meltzoff, A Bayesian Model of Imitation in Infants and Robots, Imitation and Social Learning in Robots, Humans, and Animals: Behavioural, Social and
Communicative Dimensions, K. Dautenhahn and C. Nehaniv, Eds., Cambridge University Press, 2005.

[20] C.L. Reed, What is the body schema? The Imitative Mind, A.N. Meltzoff and W. Prinz, Eds., Cambridge University Press, Cambridge, 2002, 233-243.

[21] S. Russell and P. Norvig, 2003. Artificial Intelligence a Modern Approach, Second Edition, Prentice Hall, London.

[22] P. Viviani and N. Stucchi, Biological movements look uniform, evidence for motor-perceptual interactions, Journal of Experimental Psychology, Human Perception and Performance 18(3) (1992), 603-623. 

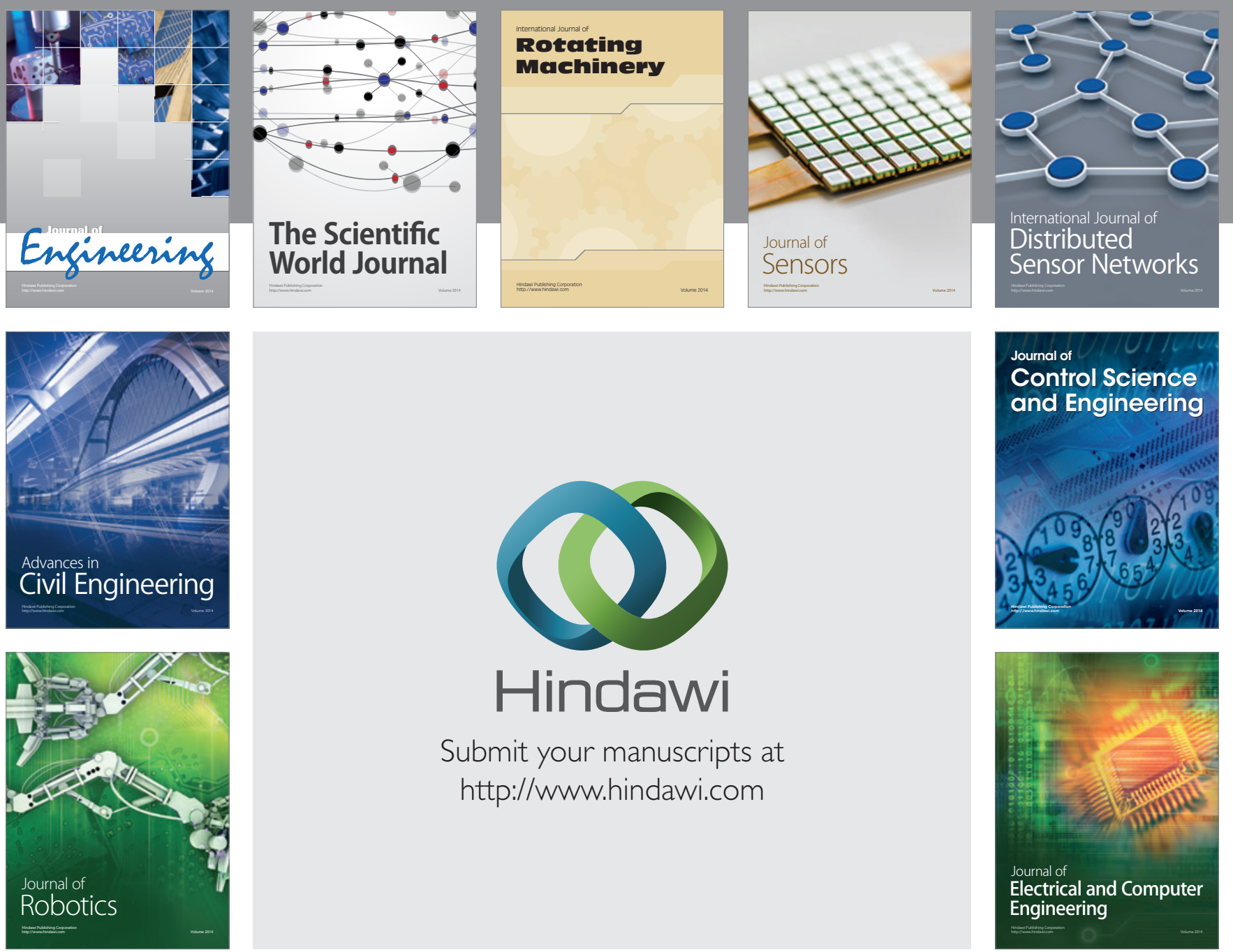

Submit your manuscripts at

http://www.hindawi.com
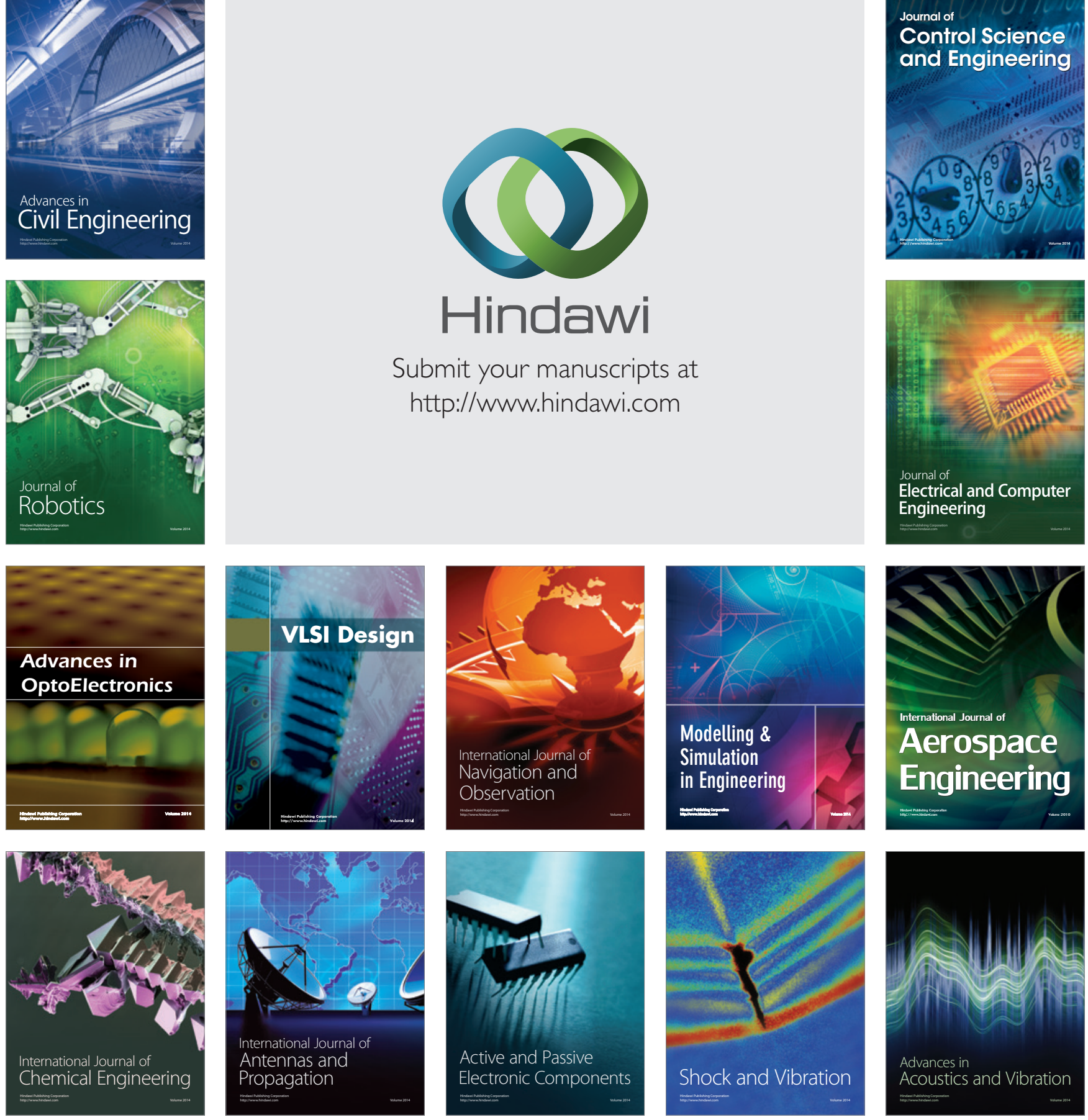